\title{
Orientaciones de un Curriculo Universitario de Competencia, desde una Perspectiva HoLÍSTICA
}

AN APPROACH TO A UNIVERSITY CURRICULUM IN COMPETENCE FROM A HOLISTIC PERSPECTIVE

Julia Romeo Cardone
Especialista en Teoría y Desarrollo del Currículo
Universidad de Chile
jromeo@uchile.cl

Resumen: El tema de las competencias en el currículo contemporáneo constituye uno de los asuntos más abordados en el mundo de hoy, tanto en Chile como en el extranjero. En este trabajo se discute el enfoque con que se opera, estimándose necesario abrirse a otras posturas, desde una perspectiva más analítica, favorecedora de contrastes que permiten tomar conciencia del significado de la(s) opción(es) por adoptar. De este modo, se plantea la existencia de orientaciones de caracteres filosofante, psicologizante, economizante y socio-politizante.

Palabras clave: currículo, competencia, holismo.

Abstract: The topic of competences in contemporary curriculum is one of the subjects most discussed in the world today, both in Chile and abroad. This article examines the approach being used in curriculum at present, considering it necessary to be open to other positions, from a more analytical perspective, thus favoring divergences to be expressed which permit becoming aware of the meaning of the options to be adopted. At the same time, the existence of philosophical, psychological, economic and socio-political approaches is also discussed.

Key words: curriculum, competence, holism.

\section{INTRODUCCIÓN}

Introducir el tópico que aquí se aborda avala el insertar algunas conclusiones finales pertinentes, del último Congreso Pedagógico Curricular efectuado en Chile, bajo la responsabilidad del Colegio de Profesores de Chile (2006).

\section{LA SOCIEDAD QUE ANHELAMOS Y LA PERSONA QUE QUEREMOS FORMAR}

Una sociedad de carácter democrático, justa y equitativa, que asuma la protección del medio ambiente y donde se valore el trabajo y la experiencia como riqueza y patrimonio de las personas. Se sustenta, en consecuencia, una sociedad participativa, solidaria y pluralista, aspirándose a una educación integral, que forme y promueva la humanización de la sociedad y, naturalmente, del ser humano que reconozcamos en ella. Una persona que esté capacitada para insertarse armónicamente en la sociedad, la familia y el trabajo, "en un mundo integrado y mundializado en lo económico, cultural tecnológico, que pueda trabajar en equipo, discernir y disentir, asumiendo la disidencia como parte de la democracia; y anteponer una visión valórica a su sentido de progreso" (op. cit., p.18). 


\section{EL SENTIDO DEL CURRÍ́CULO}

Éste apunta a "un campo socialmente construido" (op.cit., p.20), implicando -de acuerdo con lo expresado anteriormente-connotaciones políticas, económicas y culturales que permean las acciones de las agencias escolares, cualesquiera sea el nivel de su responsabilidad, favoreciendo, de un modo u otro, roles tales como los propios de un miembro de familia, de un desempeńo laboral y, sobre todo, de un ciudadano que se desenvuelve en un contexto que le corresponde.

El currículo no es neutro; es intencionado. De allí la significación de las orientaciones que se plantean y de los impactos de las decisiones que se adopten, en el sistema y en cada una de las unidades educativas donde se instalen.

Por ello, cuando entra a definirse el currículo, siempre van a surgir algunas interrogantes básicas:

¿Cuáles competencias se hacen necesarias de desarrollar?, ¿qué contenido cultural seleccionar, para favorecerlas? y ....ंcuáles estrategias de enseńanza y de aprendizaje por privilegiar?, bajo el considerando del concepto de persona y de sociedad que se esgrimen. Además de otras no menos importantes como: ¿a quiénes beneficia las opciones elegidas?, ¿¿cuáles implicancias no se han declarado, pero existen argumentos para sustentarlas o, al menos, formular hipótesis al respecto?

Desarrollar un tema como el que se anuncia no puede estar ajeno, tampoco, al concepto de calidad de la educación, puesto que cuando se definen o estipulan las competencias estimables como tales, éstas -como se tratará más adelante- estarán en relación directa con las concepciones que se sostengan.

De allí, parece significativo, y correspondiente, iniciar estas reflexiones sobre el enfoque que se presenta en María Irigoin y Fernando Varas, seńalando a Harvey (Harvey, 2000, cit. en Irigoin y Varas, 2002, p.219), y a Barnett (Barnett, 1992 y 1998, cit. en Irigoin y Varas, op. cit. supra),

Estos últimos serán, en esta ocasión, los incentivos a partir de los cuales se generen los pensamientos aquí expuestos por la autora que suscribe este artículo; así, también, cabe dar como fuente de referencia otras publicaciones de la misma (Romeo, J. y M. Llaña, 2004 a y b).

De Harvey, se destacan los cinco conceptos de calidad de la educación que él reconoce como los de mayor presencia en la literatura. La calidad como:

$1^{\circ}$ lo excepcional

$2^{\circ}$ lo perfecto

$3^{\circ}$ lo pertinente a los fines o propósitos pretendidos

$4^{\circ}$ la relación costo/beneficio

$5^{\circ}$ el desarrollo humano 
Los argumentos que se esgrimen en el análisis de las orientaciones se estiman sobre la base del tercer y del último concepto, seńalando que el primero y el segundo son muy discutibles, dadas que estas definiciones contienen un alto sesgo. El cuarto corresponde, predominantemente, a una de las orientaciones que aquí se estipulará en el momento oportuno. Y los relativos a los propósitos y al desarrollo humano son, justamente, aquellos que reafirman la postura holística que se sostiene como la más valedera.

Por otra parte, Ronald Barnett, quien actualiza el pensamiento de Burton Clark (sin referencia bibliográfica en la obra de Irigoin y Vargas que estamos utilizando como de síntesis de citas y de acotaciones del caso) apunta a los mayores ámbitos temáticos que los sistemas educacionales consideran como soporte de sus marcos de discursos, de políticas y de prácticas curriculares. Éstos son:

- là comunidad educativa

- el Estado

- el mercado

¿Qué aporta esta referencia al desarrollo de las orientaciones que se aclaran más adelante? ¡Muy simple! Si, predominantemente, funciona la comunidad como eje para la toma de decisiones, las competencias son acordadas por los actores de la comunidad académica; en este caso, de contexto universitario. Si, en cambio, es el Estado, las determinaciones emergen de los actores políticos de la sociedad, sobre la base de los estimados desempeños para afianzar el sistema imperante oficial y, si el mercado es quien selecciona, los actores empresariales son quienes eligen las competencias necesarias en función de las demandas observadas. No están ausentes, tampoco, los voceros de las instituciones doctrinarias quienes, de acuerdo con las misiones que asumen, también se incorporan a las respectivas especificaciones estimadas como deseables de desarrollar y lograr.

Así, se pretende ofrecer una fuente de reflexión sobre un tópico que se discute, con alta frecuencia, en los ámbitos universitarios en estos últimos tiempos. La influencia de modalidades extranjeras, sobre todo, las provenientes de propuestas europeas, han tenido una intensa repercusión en las reformas curriculares universitarias que se están forjando en Chile.

\section{EL CURRÍCULO Y SUS DIMENSIONES}

De hecho, en relación con el tema que aquí se aborda, emergen preguntas tales como:

- ¿Cuál es el significado que le otorgamos al término currículo para estos efectos?

- ¿Qué se entiende por competencias en el marco de la concepción de currículo que aquí se sostiene?

- ¿Es posible reconocer distintas orientaciones para el desarrollo de ellas, desde la postura que se favorece? 
A modo de respuestas aclaratorias e introductorias de un análisis más profundo, se puede seńalar lo siguiente:

Curriculo es una selección y organización de la cultura por transmitir o generar con intenciones de una evaluación contextual de los procesos y de los logros que conlleva, como desarrollo y como resultado o, en su defecto, de una situación que contempla una macro proyección, con propósitos de confrontación de dichos aspectos, a niveles más amplios que los propios del carácter local que lo individualiza.

Por consiguiente, el objeto del currículo se traduce en, por un lado, las ideas, los principios, las creencias, los postulados, los 'saberes' propios de una cultura, incluyendo en ella los conocimientos específicos de una disciplina. Por otro lado, también apunta a los quehaceres de la cultura en cuestión, traducidos en procedimientos, métodos, técnicas y formas de operar: el 'saber hacer' como se diría en otras palabras. Finalmente, abarca, inclusive, los modos de relacionarse uno con otro, las manifestaciones del mundo afectivo y de la sociedad que sostenemos como la deseable de construir o de conservar dinámicamente, si fuera el caso.

El currículo, desde la óptica que nos interesa destacar, se va a plasmar en las diferentes dimensiones (d.) que la comunidad de especialistas ha consensuado: la d. explicita (cultura pública expresada a través de planes y programas de estudio), la d. implicita (cultura interna de la institución que conforma un modo de actuar y de comportarse no declarados), una d. nula (cultura a la cual no se le ha otorgado, oficialmente, espacios para su desarrollo, correspondiendo a ausencias intencionadas).

Conclusión, el currículo es todo lo que se desarrolla intencionadamente, en una institución educativa, aun sin que los sujetos que se sitúan en ella tengan claridad de las intenciones, puesto que éstas - generalmente- responden a una filosofía y política educacional no siempre anunciada ni enunciada, en su cabalidad.

El currículo, a través de sus diversas dimensiones, constituye una vía de incorporación de cultura, ya sea en el plano de las ideofacturas, manufacturas o sociofacturas, como algunos teóricos denominarían, respectivamente, a cada uno de los aspectos vinculados a los saberes, mencionados con anterioridad. Todos son desarrollados, de un modo u otro, por las diversas intervenciones que acontecen en el ámbito de las interacciones que se dan.

\section{EL CURRÍCULO Y LAS COMPETENCIAS}

En cuanto a la competencia, definida desde un significado genérico, con -además- connotaciones filosóficas, psicológicas, económicas y sociopolíticas, se la precisa como un conjunto orgánico de conocimientos, habilidades y actitudes, que potencia desempeños estimados éstos como pertinentes, en los contextos donde ella tiene la oportunidad de demostrarse, con eficiencia y eficacia, al unísono que 
con compromiso con las tareas que implican, en el marco de una cosmovisión cultural compartida.

De allí que se sostenga que no hay selección ni organización de competencias que puedan considerarse neutras. Todas responden a la intención de asentar una ideología, en el estricto sentido del término, directa o indirectamente, aún las que se vinculan al saber hacer, las que pudieran interpretarse como operativas e instrumentales, dado que ellas -también- están al servicio de una labor, con connotaciones preconcebidas como necesarias y útiles, frente a argumentaciones esgrimibles como valederas. Con mayor razón, parecerán aceptables estos puntos de vista, si son referidos a un saber o a un saber ser, los que -de por sí- conllevan adjetivos propios de las posturas que conforman un cuerpo de conocimientos específicos o una jerarquía de valores, expresados desde la formación humana a la cual se aspira.

Si se habla de competencias, entonces, se debiera tener presente que se está enfrentando a un significado múltiple o, quizás más exactamente, a un significado de corto alcance y a otro de largo alcance. (Ver Fig. 1).

Figura 1: Orientaciones centradas en COMPETEnCias

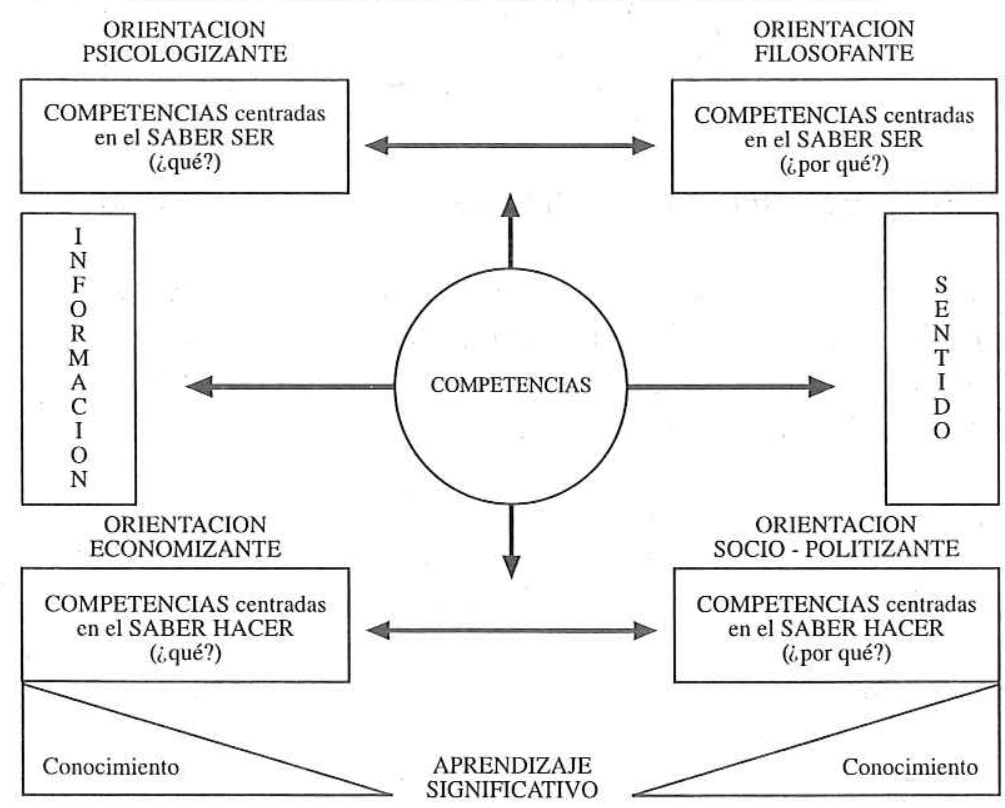

El primero, el de corto alcance, es aquél estimado con una supuesta objetividad, que ya se ha indicado como inexistente, desde una óptica de reflexión sobre la práctica curricular. El segundo, el de largo alcance, en cambio, bajo el reconocimiento que en cada competencia exigida, en el proceso de educarse, se está, al mismo tiempo, legitimando una cosmovisión de mundo, incluido en ésta, una concepción de la naturaleza del trabajo donde, entre otros ámbitos, pudiere estar predefinida la demanda de aquélla. 
Por tanto, desde este alcance, se reconocerán como competencias, no sólo distintos tipos de conocimientos, diferentes clases de habilidades y, hasta, espectros diversos de actitudes: sino -también- la anuencia frente a una variedad de énfasis, cuya síntesis podría expresarse, entre otras lecturas, de acuerdo a un criterio de figura y fondo (Ver Fig. 2).

- conocimientos, habilidades y actitudes;

- habilidades, actitudes y conocimientos;

- actitudes, conocimientos y habilidades.

Figura 2: Perspectiva atomicista

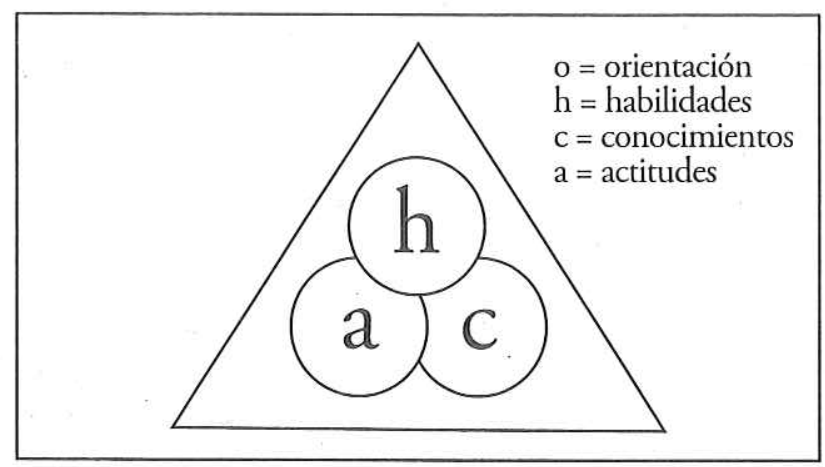

\section{LA ORIENTACIÓN HOLÍSTICA EN EL CURRÍCULO POR COMPETENCIAS}

La denominación de orientaciones, en relación a un currículo de competencias deriva, justamente, del análisis de los párrafos anteriores, puesto que se considera que no sólo se puede ofrecer una propuesta, sino -por el contrario- más de una, desde otro ángulo una de carácter global.

Para fundamentar esta postura, que se califica como holistica, en el tenor de una opción integradora, no atomística, ni excluyente, se señala que ella acepta las influencias filosóficas, psicológicas, económicas y político-sociales como criterios de selección y de organización que intervienen en la toma de decisiones de las competencias que se precisan. (Ver Fig. 3).

Figura 3: Perspectiva Holística

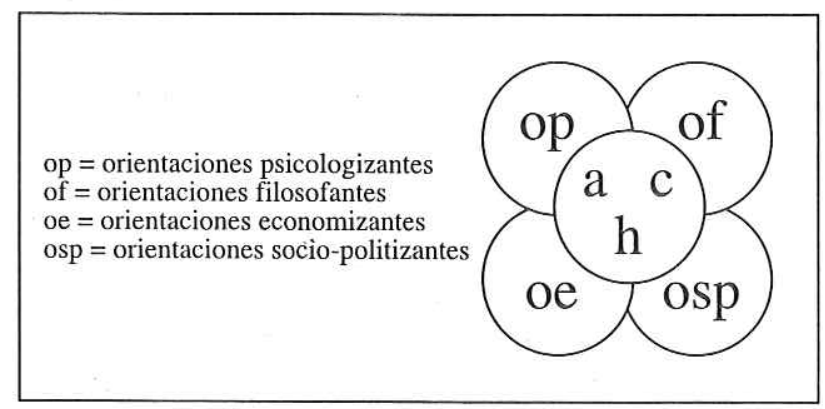


Se aclara que, en cada orientación reconocida, se proporciona un énfasis distinto, según los marcos ya mencionados, los que, indudablemente, deberán ser coherentes y consistentes entre sí. Esto último constituye un dilema que puede devenir en una propuesta confusa y contradictoria, y en un aprendizaje que no favorezca los fines educativos deseables, para constructores de sociedad.

Las competencias centradas en el 'saber ser', la orientación filosofante, considera a este saber como de imprescindible desarrollo, seleccionándose y organizándose -con preferencia- sobre la base del concepto de hombre y de mundo que subyace en la perspectiva de educación que se ha decidido como identidad profesional para el futuro.

El criterio que predomina en la gestión es aquél que da respuesta a los por qué, los que emergen en la toma de decisiones de su incorporación en el currículo. Incluso, cuando la competencia se relaciona con el desempeño en el trabajo (que no es el único horizonte al cual debe atender) -pero al cual prepara la formación profesional- éste, el desempeńo, está seńalando una preferencia por el sentido profundo de lo que se debe asumir: por los argumentos más esenciales que avalan la presencia deseada.

La orientación que corresponde a este planteamiento es una orientación de competencias centrada en los valores que se sostienen, en las actitudes que se favorecen, en los principios de vida que se portan. Por otro lado, se insiste en que la competencia no sólo se privilegia para la vida laboral, sino -también- para otros ámbitos de la vida personal y social de cada sujeto que se educa.

Desde otro ángulo, las competencias, también, favorecen un énfasis psicológico, en una orientación psicologizante, centrada en el desarrollo de rasgos de personalidad que se estima pertinente a la profesión. En este caso, el criterio que prima es el que responde al qué se requiere como modo de ser, a los cuáles son los rasgos estimados como atributos en el profesional que se prepara, ameritando una exploración fina que aporte información suficiente acerca de las condiciones personales de los sujetos que se desempeńarán con roles precisados.

La orientación economizante, difundida, generalmente, como tecnológica, y denominada también, técnica, en cambio, se sitúa desde el saber hacer, desde el compromiso que se tiene, con el estudiante, con el qué debe él (ella) dominar como procedimientos, para responder a las exigencias profesionales para las cuales la institución prepara, bajo el considerando de un criterio centrado en las ofertas y demandas del mercado, con fines de aumento de los ingresos nacionales y, como corolario de las sumas correspondientes a los particulares, a los trabajadores. (Ësta, precisamente, es la generalizada en los ámbitos curriculares, considerándola de una pura visión pragmática). 
Finalmente, en cuanto a la orientación socio-polítizante, se debe afirmar que ésta selecciona y organiza las competencias -desde un plano de figura-con las intenciones de agenciar una sociedad con determinadas características, sean por conservación o por renovación de la existente en los términos de mantener o cambiar las estructuras de poder. Por consiguiente, la determinación de ellas se sostiene, entre otros, en el sentido que se le otorga a la distribución de los ingresos de la población del país, del mundo laboral, específicamente. Preocupan el uso de estrategias pertinentes, sea en un sentido como en el otro. Se vincula con los asentamientos de los derechos y de los deberes por defender, sean de parte de los trabajadores o, en su contrapartida, de los empleadores.

En la práctica universitaria, la orientación filosofante y la socio-politizante, generalmente, no se desarrollan desde las aulas, explícitamente, sino que se insertan en el clima organizacional de la institución y, por consiguiente, se aprecian -directa o indirectamente- en el currículo implícito explicitable, o en otros casos más adoctrinantes, en el reconocido como currículo oculto (implícito no explicitable).

En síntesis, las competencias pueden precisarse desde distintas orientaciones que no son -necesariamente-- excluyentes entre sí; por el contrario, están siempre presente, de un modo u otro, cada una de ellas, en distintos planos, sea como figura $o$ como fondo.

En otras palabras, aunque la competencia incluya las dimensiones del saber, saber hacer y saber ser o, dicho de otra manera, la integración de conocimiento, habilidades y actitudes, se hace necesario tener presente que cualesquiera que sean, responden a un patrón de mundo, sociedad y persona. Por tanto, el concepto de desempeño no sólo involucra 'una preparación profesional aséptica', sino -por el contrario- involucra concepciones de vida.

De allí que todo especialista en currículo debe, cumpliendo con uno de sus roles de tal, identificar, en la selección y organización de las competencias, lo sustancial de los profundos significados que ellas encierran, la información que se socializa, el conocimiento que se hace propio y el sentido que se pretende desarrollar y lograr con los aprendizajes esperables establecidos y no establecidos.

\section{REFERENCIAS BIBLIOGRAFICAS}

Colegio de Profesores de Chile A.G., 2006. Conclusiones Finales Congreso Curricular 2005. En: Docencia, No 28, Colegio de Profesores de Chile AG, mayo. Santiago. Pp.4-39.

Irigoin, M. y F. Varas, 2002.

Competencias Laborales, OIT, CINTERFOR Montevideo, 250 pp. 
Romeo, J., у M. Llaña, 2004 a.

Romeo, J., y M. Llańa, y Colaboradoras, $2004 \mathrm{~b}$.
Hacia una construcción de una teoría comprensivo-interpretativa de currículo. Revista Enfoques Educacionales, Santiago 6 (1). $131-142$.

En busca del concepto de solidaridad que manejan nuestros adolescentes. Primeras aproximaciones. Vicerrectoría de Asuntos Académicos, Departamento de Investigaciones y Desarrollo. Escuela de Postgrado de la FACSO, Universidad de Chile, Ediciones M. G. Comunicaciones Cantero y Gómez, Ltda., Santiago de Chile. 200 pp. 